\title{
Exponential Functions for Modelling Hysteresis of Soil-Water Characteristic Curves
}

\author{
Alfrendo Satyanaga ${ }^{1, *}$, Jong $\mathrm{Kim}^{2}$, Sung-Woo Moon $^{3}$ and Martin Wijaya ${ }^{4}$ \\ ${ }^{1}$ Assistant Professor, Department of Civil and Environmental Engineering, Nazarbayev University, 53 Kabanbay Batyr Ave, 010000 , \\ Nur-Sultan, Kazakhstan \\ ${ }^{2}$ Professor, Department of Civil and Environmental Engineering, Nazarbayev University, 53 Kabanbay Batyr Ave, 010000, Nur-Sultan, \\ Kazakhstan \\ ${ }^{3}$ Assistant Professor, Department of Civil and Environmental Engineering, Nazarbayev University, 53 Kabanbay Batyr Ave, 010000 , \\ Nur-Sultan, Kazakhstan \\ ${ }^{4}$ Lecturer, Parahyangan Catholic University, Jl. Ciumbuleuit No. 94 Bandung, Indonesia
}

\begin{abstract}
Soil - water characteristic curve (SWCC) is an important property of unsaturated soils that can be used to estimate various parameters to describe unsaturated soil behavior. SWCC is reported to be hysteretic because the water content at a given suction in the wetting process is less than that in the drying process. In order to simulate the hysteretic characteristics of SWCC, many models have been proposed by different researchers. However, majority of the existing models are complex and their parameters are not related to the physical significances of SWCC variables. In this study, the new equations are developed to model drying and wetting SWCC. In addition, some indexes are proposed to estimate the wetting SWCC from drying SWCC. The new equations for SWCCs were evaluated with the laboratory data from published literatures. The results showed that the proposed equations performed well in modelling drying and wetting SWCC. The new equation has less parameters than the existing published equation.
\end{abstract}

\section{Introduction}

Soil - water characteristic curve (SWCC) is an important property of unsaturated soils which can be used to estimate the permeability and shear strength of the unsaturated soil. These unsaturated soil properties are required to address many geotechnical problems. Since SWCC is used as the basis for the estimation of other unsaturated soil parameters, it is important to have a mathematical model which fitting parameters can be used to represent the variables of SWCC. Numerous equations have been suggested to model the experimental data of SWCC [1, 2, 3, 4, 5, 6]. However, these equations were limited for the drying SWCC.

The hysteretic nature of SWCC has been known for a long time and it can be attributed to multiple factors such as "ink-bottle" effect, contact angle difference during the drying and wetting processes, entrapped air and thixotropic regain due to drying and wetting history of the soil [7]. Bear [8] demonstrated that the hysteresis of SWCC is resulted from "ink-bottle" effect and "raindrop" effect, which means that the contact angle at an advancing interface in the wetting process is different from that at a receding interface in the drying process. Assuming the soil pores are connecting to each other, the capillary water rise height is shorter in the wetting process than that in the drying process. This phenomenon is called "ink-bottle" effect.
Many models have been proposed by different researchers to simulate the drying and wetting curve of SWCC $[9,10,11,12,13,14]$. However, their mathematical equations are complex and their parameters are not related to the physical significances of SWCC variables. Therefore, in this paper, a new equation to model drying and wetting SWCC is proposed. In addition, the new method to predict the wetting SWCC from the drying SWCC using the proposed equation is also provided in this paper.

\section{Theory}

SWCC is defined as the relationship between water content and suction of a soil $[15,16]$. The water content defines the amount of water contained within the pores of the soil. Volumetric water content, $\theta_{\mathrm{w}}$ (volume of water in soil divided by total volume of soil, or $\left.V_{w} / V\right)$ is most commonly used. The suction used is matric suction, $\psi$ (i.e. $\mathrm{u}_{\mathrm{a}}-\mathrm{u}_{\mathrm{w}}$, where $\mathrm{u}_{\mathrm{a}}$ is the pore air pressure and $\mathrm{u}_{\mathrm{w}}$ is the pore water pressure). SWCC is usually plotted in a semi-logarithmic scale, i.e. volumetric water content is in arithmetic scale, and matric suction is in logarithmic scale.

The air-entry value, $\psi_{\text {aev }}$ of a soil is the matric suction where air first enters the largest pores in the soil. The residual water content, $\theta_{\mathrm{r}}$, is the water content where the slope of the SWCC decreases considerably and a 
large suction change is required to remove additional water from the soil. The suction corresponding to zero water content appears to be the same for all types of soil. For a wide range of soil, a value of $10^{6} \mathrm{kPa}$ has been experimentally supported [15]. In other words, the maximum matric suction to be considered in the SWCC is $10^{6} \mathrm{kPa}$ for all types of soil.

Many empirical equations have been proposed to best fit the SWCC. One of the earliest equations was proposed by Brooks and Corey [1]. Their equation was found to perform quite well for suction ranges greater than the air-entry value of the soil. However, it did not fit well under a fully saturated condition or near maximum desaturation. Another frequently used equation for SWCC was given by van Genuchten [2]. In an attempt to obtain a closed-form equation for permeability, van Genuchten [2] suggested the relationship $m=1-1 / n$. However, this restricts the flexibility of the equation. The equation would perform better without $\mathrm{m}$ and $\mathrm{n}$ having any fixed relationship. Fredlund and Xing [6] proposed a four-parameter equation to best fit SWCC. Their equation has been proven to provide better fit than other equations mentioned above [17]. However, the parameters of their equation are not related to the variables of drying and wetting SWCC.

Pham et al. [12] proposed a simple scaling method to predict the boundary wetting curve entirely from the boundary drying curve. The curve-fitting parameters of the boundary wetting curve can be calculated using fitting parameters of the boundary drying curve, together with the distance, and slope ratio between the two boundary curves. Pham et al. [12] suggested several values for the slope ratio and the distance between the two boundary curves for different types of soil. It has been shown that the scaling method provides acceptable prediction of the boundary wetting curve. However, this method still uses the equation which parameters do not take into account the physical significance of its parameters.

\section{Proposed Equation}

The proposed equations for modelling SWCC in this study have the form of exponential functions, and each fitting parameter has a physical meaning related to the fitting curve. The equations were derived based on the consideration that the shape of SWCC is similar to the graph of the exponential function (Figure 1). It can be seen that the values of the exponential function in Figure 1 are within the range of 0 to 1 . Therefore, the equation for SWCC can be expressed as:

$$
\Theta=\exp [-F(\psi)]
$$

where :

$\Theta=\frac{\theta-\theta_{r}}{\theta_{s}-\theta_{r}}=$ normalized volumetric water content
$\mathrm{F}(\psi)=$ a function of matric suction, whose parameters are properties of the SWCC such as the air-entry value, $\psi_{\text {aev }}$, and residual matric suction, $\psi_{\mathrm{r}}$.

$$
F(\psi)=\left\{\begin{array}{cl}
0 & \text { for } \psi<\psi_{\mathrm{aev}} \\
5\left(\frac{\psi-\psi_{\text {aev }}}{\psi_{r}-\psi_{\text {aev }}}\right) & \text { for } \psi \geq \psi_{\mathrm{aev}}
\end{array}\right.
$$

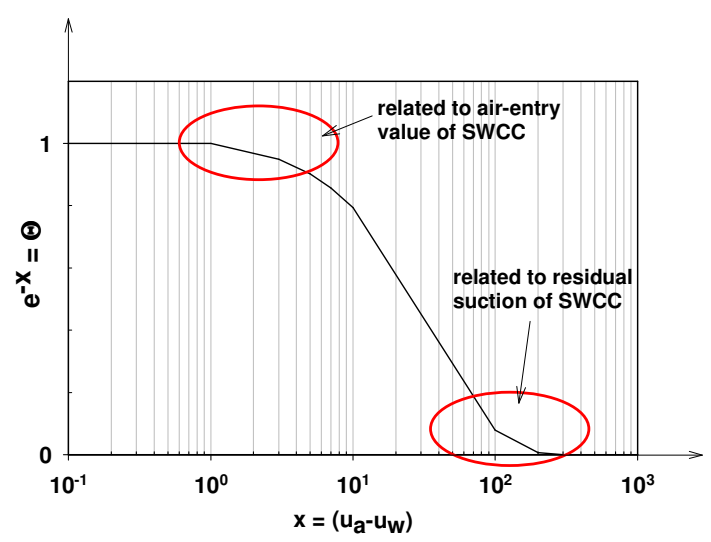

Fig. 1. Graph of exponential function $f(x)=\exp (-x)$

The Fredlund and Xing [6] correction factor was applied to the equation. The volumetric water content at any matric suction is calculated from the equation 4 . The initial value of each fitting parameter can be approximated from the variables of the SWCCs as obtained from laboratory tests and illustrated in Figure 2. An iterative non-linear regression procedure can be used to obtain the final values of each fitting parameters. Regardless of the approximations of the initial values, the final values of each fitting parameter will be the same, demonstrating the uniqueness of the solution to this equation.

$$
\begin{aligned}
& \theta(\psi)=\left[1-\frac{\ln \left(1+\frac{\psi}{\psi_{r}}\right)}{\ln \left(1+\frac{10^{6}}{\psi_{r}}\right)}\right]\left\{\left(\theta_{s}-\right.\right. \\
& \left.\left.\theta_{r}\right) \exp \left[-5\left(\frac{\psi-\psi_{a e v}}{\psi_{r}-\psi_{a e v}}\right)\right]+\theta_{r}\right\}
\end{aligned}
$$

where:

$\theta_{\mathrm{s}}=$ saturated volumetric water contents

$\theta_{\mathrm{r}}=$ residual volumetric water contents

$\psi_{\text {aev }}=$ air-entry value of soil

$\psi_{\mathrm{r}}=$ residual matric suction

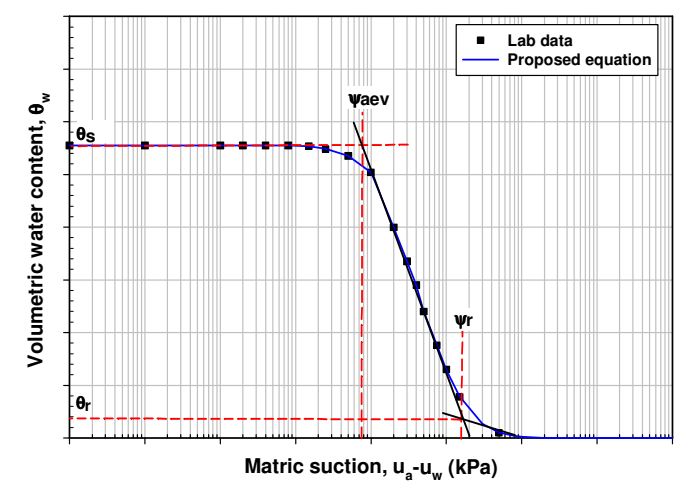

Fig. 2. Approximation of initial values for best fit parameters of SWCC 
For wetting SWCC, the equation has the following form:

$$
\begin{aligned}
& \theta(\psi)=\left[1-\frac{\ln \left(1+\frac{\psi}{\psi_{w}}\right)}{\ln \left(1+\frac{10^{6}}{\psi_{r w}}\right)}\right]\left\{\left(\theta_{s}-\right.\right. \\
& \left.\left.\theta_{r}\right) \exp \left[-5\left(\frac{\psi-\psi_{b w}}{\psi_{w}-\psi_{b w}}\right)\right]+\theta_{r}\right\}
\end{aligned}
$$

where:

$\psi_{\mathrm{bw}}=$ wetting saturation point (expulsion value) of SWCC

$\psi_{\mathrm{w}}=$ water-entry value of SWCC

The wetting saturation point and water-entry value of SWCC are related to the air-entry value and residual matric suction of the SWCC by the following expressions:

$$
\begin{gathered}
\psi_{b w}=\chi_{a e v} \psi_{a e v} \\
\psi_{w}=\chi_{r} \psi_{r}
\end{gathered}
$$

where:

$\chi_{\mathrm{aev}}$ and $\chi_{\mathrm{r}}=$ parameters related to the hysteresis in SWCC

In Equations 5, 6 and 7, the exponential functions are to take value of 1 if the value of the exponent is positive.

\section{Evaluation of the proposed equation and estimation of wetting SWCC}

A total of 12 soil data were used for evaluation of fitting drying and wetting SWCCs which were taken from

\begin{tabular}{|c|c|c|c|c|}
\hline Soil type & Source & $\begin{array}{l}\text { Void } \\
\text { ratio, } \mathrm{e}\end{array}$ & $\begin{array}{c}\text { Dry } \\
\text { density, } \rho_{\mathrm{d}} \\
\left(\mathrm{Mg} / \mathrm{m}^{3}\right) \\
\end{array}$ & $\begin{array}{c}\text { Specific } \\
\text { gravity, Gs }\end{array}$ \\
\hline $\begin{array}{l}\text { Beaver Creek } \\
\text { Sand } \\
\end{array}$ & \multirow[t]{2}{*}{$\begin{array}{c}\text { Pham et al. } \\
\text { (2003) }\end{array}$} & 0.563 & 1.70 & 2.65 \\
\hline Processed silt & & 0.689 & 1.58 & 2.67 \\
\hline Soil mixture & \multirow{2}{*}{$\begin{array}{c}\text { Indrawan } \\
\text { et al. } \\
\text { (2007) }\end{array}$} & 0.510 & 1.74 & 2.63 \\
\hline Gravelly sand I & & 0.640 & 1.62 & 2.66 \\
\hline Gravelly sand II & $\begin{array}{c}\text { Yang et al. } \\
(2004)\end{array}$ & 0.617 & 1.62 & 2.62 \\
\hline Compacted silt & \multirow{2}{*}{$\begin{array}{c}\text { Trinh et al. } \\
(2006)\end{array}$} & 1.083 & 1.35 & 2.65 \\
\hline Loose silty sand & & 0.677 & 1.61 & 2.70 \\
\hline Dense silty sand & \multirow{2}{*}{$\begin{array}{c}\text { Pham et al. } \\
(2003)\end{array}$} & 0.424 & 1.88 & 2.68 \\
\hline Caribou silt loam & & 0.655 & 1.61 & 2.67 \\
\hline Medium sand & \multirow{2}{*}{$\begin{array}{c}\text { Yang et al. } \\
(2004)\end{array}$} & 0.538 & 1.69 & 2.60 \\
\hline Fine sand & & 0.699 & 1.56 & 2.65 \\
\hline Coarse kaolin & $\begin{array}{l}\text { Meilani et } \\
\text { al. (2005) }\end{array}$ & - & - & - \\
\hline
\end{tabular}
different published literatures [11, 18, 19, 20, 21]. Basic properties of those soils are summarized in Table 1.

Table 1. Basic properties of the data used in this study

For verification of the accuracies of the proposed equations, a nonlinear least squares optimization procedure was used to analyze the laboratory data. The procedure was performed using an iterative nonlinear regression procedure that is provided in the Microsoft Excel software [22]. The coefficient of determination $\left(\mathrm{R}^{2}\right)$ of the fitted curves versus the laboratory data were used to evaluate the accuracies of the proposed equations.

The coefficient of determination $\left(\mathrm{R}^{2}\right)$ and values of fitting parameters of each best fit curve are presented in
Tables 2 and 3 . Tables 2 and 3 show that the coefficients of determination, $\mathrm{R}^{2}$, of all best fit curves are very close to unity, with the lowest $\mathrm{R}^{2}$ being above 0.96 . The average values of $\mathrm{R}^{2}$ are 0.997 for drying SWCC and 0.991 for wetting SWCC. Therefore, both equations performed well in fitting data of SWCC. Moreover, the fitting parameters are closely related to the variables of SWCC. The fitted values of $\psi_{\mathrm{bw}}$ and $\psi_{\mathrm{w}}$ are approximately equal to the wetting saturated suction and water-entry value of the SWCC. This can be verified from the graphs of the fitted curves. Figures 3 to 5 show the best fit curves for the soils: processed silt, gravelly sand I, and dense silty sand, respectively. However, the values of two parameters $\left(\chi_{\mathrm{bw}}\right.$ and $\left.\chi_{\mathrm{w}}\right)$ in each best fit curve were not predicted from the drying SWCC, but they were fitting parameters. Therefore, it is necessary to have some kind of expressions to calculate $\chi_{\mathrm{bw}}$ and $\chi_{\mathrm{w}}$ or some proposed values of $\chi_{b w}$ and $\chi_{w}$ for different soil types for complete prediction of boundary wetting SWCC from boundary drying SWCC. For this purpose, the two parameters $\left(\chi_{\mathrm{bw}}\right.$ and $\left.\chi_{\mathrm{w}}\right)$ have been related to two parameters: the slope ratio, RSL, and distance between the two boundary curves, DSL, as mentioned in Pham et al. (2005). A schematic illustration of the relationship between the parameters of the two curves is shown in Figure 6.

Table 2. Values of Fitting Parameters and Coefficient of Determination $\left(\mathrm{R}^{2}\right)$ of drying SWCC

\begin{tabular}{|c|c|c|c|c|c|}
\hline \multirow{2}{*}{ Soil } & \multicolumn{5}{|c|}{ Drying SWCC (Equation 4) } \\
\cline { 2 - 6 } & $\begin{array}{c}\boldsymbol{\Psi}_{\text {aev }} \\
(\mathbf{k P a})\end{array}$ & $\begin{array}{c}\boldsymbol{\psi}_{\mathbf{r}} \\
(\mathbf{k P a})\end{array}$ & $\boldsymbol{\theta}_{\mathbf{s}}$ & $\boldsymbol{\theta}_{\mathbf{r}}$ & $\mathbf{R}^{\mathbf{2}}$ \\
\hline \hline $\begin{array}{c}\text { Beaver Creek } \\
\text { Sand }\end{array}$ & 1.6 & 18 & 0.361 & 0.119 & 0.992 \\
\hline Processed silt & 6.2 & 269 & 0.407 & 0.131 & 0.998 \\
\hline Soil mixture & 0.10 & 13 & 0.334 & 0.185 & 0.992 \\
\hline Gravelly sand I & 0.06 & 0.61 & 0.377 & 0.018 & 0.998 \\
\hline Gravelly sand II & 0.12 & 0.94 & 0.380 & 0.024 & 0.999 \\
\hline Compacted silt & 36 & 583 & 0.520 & 0.129 & 0.999 \\
\hline Loose silty sand & 2.5 & 7.9 & 0.408 & 0.033 & 0.999 \\
\hline Dense silty sand & 2.8 & 8.9 & 0.467 & 0.052 & 0.999 \\
\hline Caribou silt loam & 7.6 & 41 & 0.398 & 0.306 & 0.999 \\
\hline Medium sand & 0.81 & 9.1 & 0.350 & 0.074 & 0.996 \\
\hline Fine sand & 1.4 & 11 & 0.411 & 0.025 & 0.996 \\
\hline Coarse kaolin & 42 & 617 & 0.519 & 0.073 & 0.999 \\
\hline
\end{tabular}

Table 3. Values of Fitting Parameters and Coefficient of Determination $\left(\mathrm{R}^{2}\right)$ of wetting SWCC

\begin{tabular}{|c|c|c|c|c|c|}
\hline \multirow{2}{*}{ Soil } & \multicolumn{5}{|c|}{ Wetting SWCC (Equation 5) } \\
\cline { 2 - 6 } & $\begin{array}{c}\boldsymbol{\Psi}_{\text {bw }} \\
(\mathbf{k P a})\end{array}$ & $\begin{array}{c}\boldsymbol{\Psi}_{\mathbf{w}} \\
(\mathbf{k P a})\end{array}$ & $\boldsymbol{\chi}_{\text {aev }}$ & $\boldsymbol{\chi}_{\mathbf{r}}$ & $\mathbf{R}^{2}$ \\
\hline \hline $\begin{array}{c}\text { Beaver Creek } \\
\text { Sand }\end{array}$ & 0.88 & 9.4 & 0.55 & 0.52 & 0.998 \\
\hline Processed silt & 3.04 & 110 & 0.49 & 0.41 & 0.997 \\
\hline Soil mixture & 0.09 & 1.6 & 0.90 & 0.12 & 0.964 \\
\hline Gravelly sand I & 0.016 & 0.45 & 0.26 & 0.74 & 0.997 \\
\hline Gravelly sand II & 0.001 & 0.46 & 0.01 & 0.49 & 0.991 \\
\hline Compacted silt & 14 & 315 & 0.40 & 0.54 & 0.998 \\
\hline Loose silty sand & 0.85 & 4.98 & 0.34 & 0.63 & 0.998 \\
\hline Dense silty sand & 0.868 & 6.0 & 0.31 & 0.67 & 0.998 \\
\hline Caribou silt loam & 2.356 & 27 & 0.31 & 0.66 & 0.981 \\
\hline Medium sand & 0.1458 & 4.2 & 0.18 & 0.46 & 0.980 \\
\hline Fine sand & 0.87 & 4.5 & 0.62 & 0.41 & 0.996 \\
\hline Coarse kaolin & 11 & 413 & 0.25 & 0.67 & 0.998 \\
\hline
\end{tabular}




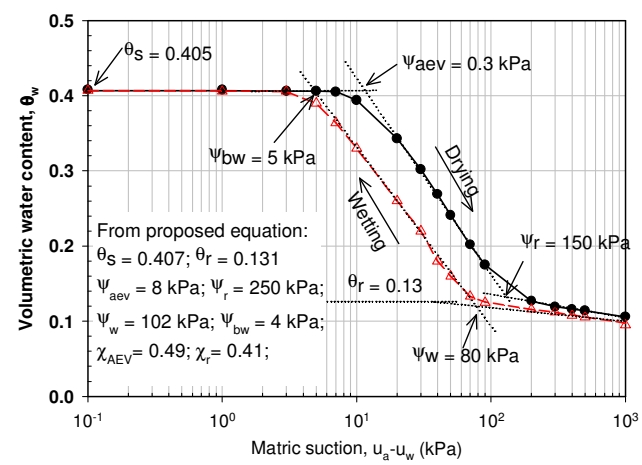

Fig. 3. Best fit curves of drying and wetting SWCC for the soil processed silt

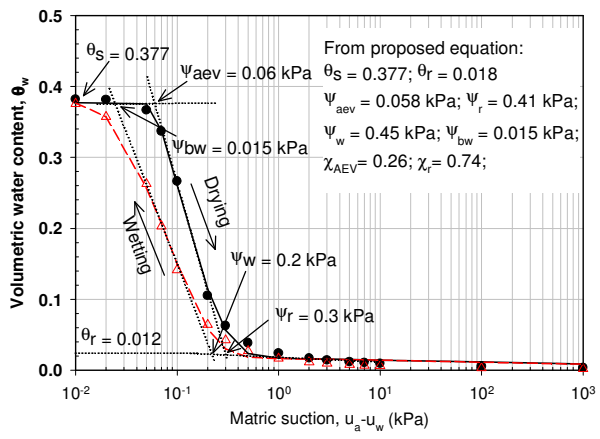

Fig. 4. Best fit curves of drying and wetting SWCC for the soil gravelly sand I

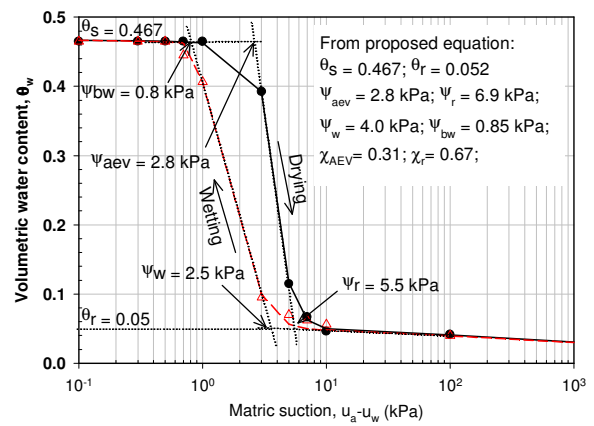

Fig. 5. Best fit curves of drying and wetting SWCC for the soil dense silty sand

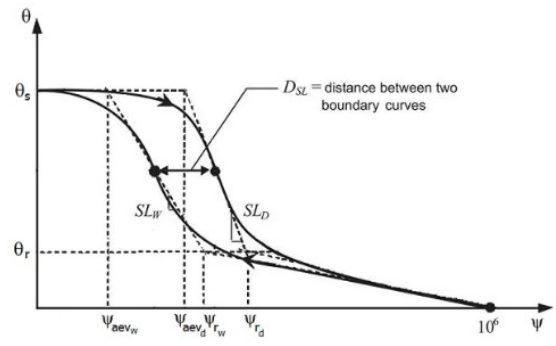

Fig. 6. A schematic illustration of the slope and distance between the two boundary curves (modified after Pham et al. [12])

The slopes of the boundary drying SWCC and boundary wetting SWCC can be calculated as:

$$
\begin{aligned}
& S L_{D}=\frac{\theta_{S}-\theta_{r}}{\log \psi_{r}-\log \psi_{a e v}}=\frac{\theta_{S}-\theta_{r}}{\log \frac{\psi_{r}}{\psi_{a e v}}} \\
& S L_{w}=\frac{\theta_{S}-\theta_{r}}{\log \psi_{w}-\log \psi_{b w}}=\frac{\theta_{S}-\theta_{r}}{\log \frac{\psi_{w}}{\psi_{b w}}}
\end{aligned}
$$

The slope ratio $\mathrm{R}_{\mathrm{SL}}$ can be expressed as:

$R_{S L}=\frac{S L_{D}}{S L_{w}}=\frac{\log \frac{\psi_{w}}{\psi_{b w}}}{\log \frac{\psi_{r}}{\psi_{\text {aev }}}}=\frac{\log \frac{\chi_{r} \psi_{r}}{\chi_{a e v} \psi_{a e v}}}{\log \frac{\psi_{r}}{\psi_{a e v}}}=1+\frac{\log \frac{\chi_{r}}{\chi_{a e v}}}{\log \frac{\psi_{r}}{\psi_{a e v}}}$

Let $\lambda$ be the logarithm of the ratio between residual matric suction and air-entry value of the boundary drying SWCC:

$$
\lambda=\log \frac{\psi_{r}}{\psi_{\text {aev }}}
$$

Equation 11 can be rewritten to obtain:

$$
\log \frac{\chi_{r}}{\chi_{\text {aev }}}=\lambda\left(R_{S L}-1\right)
$$

The ratio between $\chi_{\mathrm{r}}$ and $\chi_{\text {aev }}$ can now be written as:

$$
\frac{\chi_{r}}{\chi_{a e v}}=10^{\lambda\left(R_{S L^{-1}}\right)}
$$

It is reasonable to assume that the distance between the two boundary curves can be estimated as the average of the distance between the air-entry values to the wetting saturated point $\left(\mathrm{D}_{\text {aev }}\right)$ and the distance between the residual matric suctions to the water-entry value $\left(D_{r}\right)$ of the drying and wetting curves:

$$
D_{S L}=\frac{1}{2}\left(D_{a e v}+D_{r}\right)
$$

$\mathrm{D}_{\mathrm{aev}}$ and $\mathrm{D}_{\mathrm{r}}$ can be calculated as:

$$
\begin{gathered}
D_{a e v}=\log \psi_{a e v}-\log \psi_{b w}=\log \frac{\psi_{a e v}}{\psi_{b w}}=\log \frac{1}{\chi_{a e v}} \\
D_{r}=\log \psi_{r}-\log \psi_{w}=\log \frac{\psi_{r}}{\psi_{w}}=\log \frac{1}{\chi_{r}}
\end{gathered}
$$

Equation 14 can be rewritten as:

$$
D_{S L}=\frac{1}{2}\left(\log \frac{1}{\chi_{a e v}}+\log \frac{1}{\chi_{r}}\right)=\frac{1}{2} \log \left(\frac{1}{\chi_{a e v} \chi_{r}}\right)
$$

Equation 17 can be rearranged as follows:

$$
\chi_{a e v} \chi_{r}=10^{-2 D_{S L}}
$$

Combining Equation 14 and 18, $\chi_{\text {aev }}$ and $\chi_{\mathrm{r}}$ can be calculated as follows:

$$
\begin{gathered}
\chi_{a e v}=10\left[-D_{S L}-\frac{1}{2} \lambda\left(R_{S L}-1\right)\right] \\
\chi_{r}=10\left[-D_{S L}+\frac{1}{2} \lambda\left(R_{S L}-1\right)\right]
\end{gathered}
$$

From Equations 18 and 19, two parameters $\left(\chi_{\text {aev }}\right.$ and $\chi_{\mathrm{r}}$ ) can be calculated from the slope ratio, RSL, distance between the two curves, DSL, and the ratio between residual matric suction and air-entry value of the boundary drying curve, $\lambda$. Pham et al. (2005) suggested different values of DSL and RSL for different types of 
soil, as summarized in Table 6. These values can be used to calculate $\chi_{\text {aev }}$ and $\chi_{\mathrm{r}}$, and therefore the boundary wetting SWCC can be completely predicted from the boundary drying SWCC. Comparisons of the $\mathrm{R}^{2}$ of the predicted wetting curves between this method and Pham et al. [12] scaling method are presented in Tables 4 and 5 , respectively.

Table 4. $\mathrm{R}^{2}$ of the proposed method in predicting of wetting SWCC

\begin{tabular}{|c|c|c|c|c|c|}
\hline Soil & \multirow{2}{*}{ DSL } & \multirow{2}{*}{ RSL } & \multicolumn{3}{|c|}{ Proposed method } \\
\cline { 4 - 6 } & & & $\boldsymbol{\chi}_{\text {aev }}$ & $\boldsymbol{\chi}_{\mathbf{r}}$ & $\mathbf{R}^{2}$ \\
\hline \hline Beaver Creek Sand & 0.35 & 1.0 & 0.45 & 0.45 & 0.988 \\
\hline Processed silt & 0.35 & 1.0 & 0.45 & 0.45 & 0.996 \\
\hline Soil mixture & 0.35 & 1.0 & 0.45 & 0.45 & 0.865 \\
\hline Gravelly sand I & 0.20 & 2.0 & 0.19 & 1.0 & 0.983 \\
\hline Gravelly sand II & 0.20 & 2.0 & 0.22 & 1.0 & 0.725 \\
\hline Compacted silt & 0.35 & 1.0 & 0.45 & 0.45 & 0.993 \\
\hline Loose silty sand & 0.20 & 2.0 & 0.35 & 1.0 & 0.969 \\
\hline Dense silty sand & 0.20 & 2.0 & 0.35 & 1.0 & 0.978 \\
\hline Caribou silt loam & 0.50 & 1.5 & 0.21 & 0.48 & 0.873 \\
\hline Medium sand & 0.20 & 2.0 & 0.19 & 1.0 & 0.926 \\
\hline Fine sand & 0.20 & 2.0 & 0.22 & 1.0 & 0.959 \\
\hline Coarse kaolin & 0.50 & 1.5 & 0.16 & 0.62 & 0.992 \\
\hline
\end{tabular}

Table 5. $\mathrm{R}^{2}$ of Pham et al. [12] scaling method in predicting wetting SWCC

\begin{tabular}{|c|c|c|c|c|c|c|}
\hline Soil & \multirow{2}{*}{ DSL } & \multirow{2}{*}{ RSL } & \multicolumn{4}{|c|}{ Proposed method } \\
\cline { 4 - 7 } & & & $\mathbf{b}_{\mathbf{w}}$ & $\mathbf{c}_{\mathbf{w}}$ & $\mathbf{d}_{\mathbf{w}}$ & $\mathbf{R}^{\mathbf{2}}$ \\
\hline \hline Beaver Creek Sand & 0.35 & 1.0 & 2.82 & 0.112 & 1.94 & 0.969 \\
\hline Processed silt & 0.35 & 1.0 & 131.8 & 0.134 & 1.65 & 0.996 \\
\hline Soil mixture & 0.35 & 1.0 & 0.49 & 0.202 & 1.19 & 0.868 \\
\hline Gravelly sand I & 0.20 & 2.0 & 0.06 & 0.015 & 1.17 & 0.967 \\
\hline Gravelly sand II & 0.20 & 2.0 & 0.07 & 0.015 & 1.39 & 0.751 \\
\hline Compacted silt & 0.35 & 1.0 & 9746 & 0.121 & 2.36 & 0.989 \\
\hline Loose silty sand & 0.20 & 2.0 & 11.9 & 0.040 & 3.46 & 0.954 \\
\hline Dense silty sand & 0.20 & 2.0 & 20.1 & 0.056 & 3.32 & 0.973 \\
\hline Caribou silt loam & 0.50 & 1.5 & 132.9 & 0.328 & 3.67 & 0.828 \\
\hline Medium sand & 0.20 & 2.0 & 1.37 & 0.077 & 1.30 & 0.920 \\
\hline Fine sand & 0.20 & 2.0 & 2.63 & 0.051 & 1.96 & 0.949 \\
\hline Coarse kaolin & 0.50 & 1.5 & 1870 & 0.084 & 2.10 & 0.865 \\
\hline
\end{tabular}

From Tables 4 and 5, it can be observed that the coefficients of determination, $\mathrm{R}^{2}$, of most of the predicted curves are quite close to unity for both methods. Thus, the proposed method performed well in predicting wetting SWCC from drying SWCC. Figures 7 to 9 show the graphs of predicted wetting SWCCs for the soils: Beaver Creek sand, compacted silt, and coarse kaolin, respectively. In addition, the fitting parameters of the proposed equation for drying and wetting SWCC are closely related to the variables of the SWCC, such as airentry value, and residual matric suction. The hysteresis effect was characterized by two parameters, $\chi_{\mathrm{ave}}$ and $\chi_{\mathrm{r}}$, which are the ratio of the air-entry values of SWCC to the wetting saturation point of SWCC, and ratio of the residual matric suctions of SWCC to the water-entry value of SWCC, respectively. For simplicity, values of $\chi_{\text {ave }}$ and $\chi_{\mathrm{r}}$ are suggested for several types of soil, as shown in Table 6.

Table 6. Suggested value of $\chi_{\text {ave }}$ and $\chi_{\mathrm{r}}$ for several types of soil

\begin{tabular}{|c|c|c|c|c|}
\hline Soil type & \multicolumn{2}{|c|}{$\chi_{\text {aev }}$} & \multicolumn{2}{c|}{$\chi_{\mathrm{r}}$} \\
\hline & Range & $\begin{array}{c}\text { Typical } \\
\text { value }\end{array}$ & Range & $\begin{array}{c}\text { Typical } \\
\text { value }\end{array}$ \\
\hline \hline Graded sand & $0.10-0.30$ & 0.20 & $0.40-0.80$ & 0.60 \\
\hline $\begin{array}{c}\text { Silty sand and } \\
\text { clayey sand }\end{array}$ & $0.20-0.50$ & 0.35 & $0.60-0.80$ & 0.70 \\
\hline $\begin{array}{c}\text { Silt loam and clay } \\
\text { loam }\end{array}$ & $0.20-0.40$ & 0.30 & $0.40-0.70$ & 0.55 \\
\hline Compacted silt & $0.40-0.60$ & 0.50 & $0.40-0.60$ & 0.50 \\
\hline
\end{tabular}

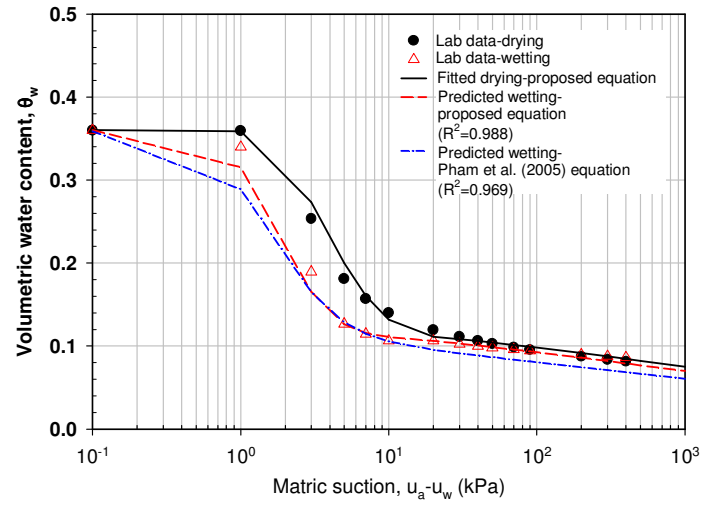

Fig. 7. Experimental data and predicted wetting SWCC for the soil Beaver Creek sand

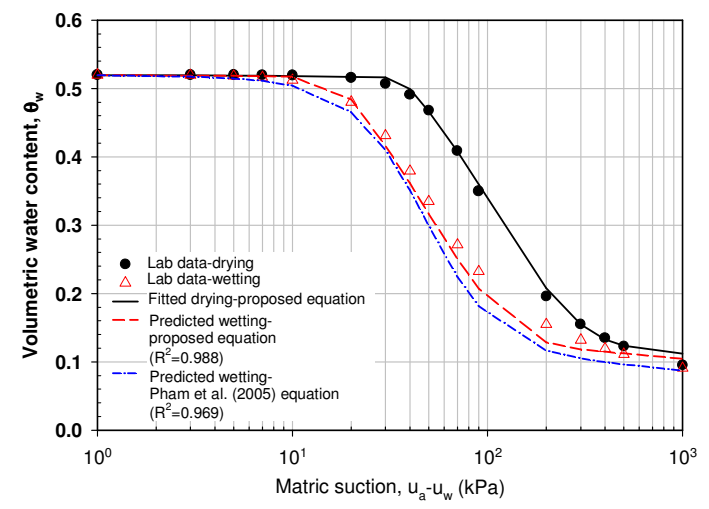

Fig. 8. Experimental data and predicted wetting SWCC for the soil compacted silt

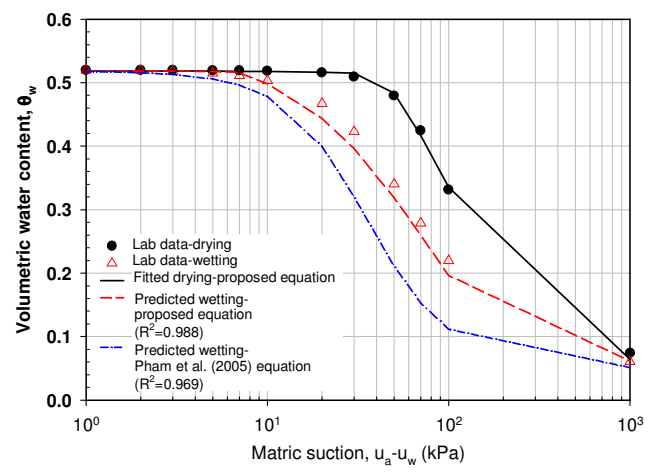

Fig. 9. Experimental data and predicted wetting SWCC for the soil coarse kaolin

\section{Conclusions}

A mathematical model of SWCC has been developed and verified with experimental data. The equations were proposed for modelling drying and wetting SWCC. In addition, a new method was also proposed to predict wetting SWCC from drying SWCC. By comparison with the published experimental data, the proposed equations have been proved to provide a good fit for drying and wetting SWCC. The proposed method for predicting wetting SWCC has been also proven to provide good prediction of wetting SWCC for different types of soils. The fitting parameters of the proposed equations closely represent the physical properties of the fitting curves, 
which can be used to describe the entire best fit curve of SWCC. The procedure of best fitting SWCC can also be shortened since the fitting parameters can be readily approximated from experimental data.

\section{Acknowledgements}

This work was supported by a research grant (SOE2017003) from Nazarbayev University. The authors gratefully acknowledge the support from staffs in School of Civil and Environmental Engineering, Nazarbayev University during the data collections.

\section{References}

1. R.H. Brooks and A.T. Corey, Hydrology Paper, 3, Colorado State University, USA (1964).

2. M.T. Van Genuchten, Soil Science Society of America Journal, 48: 142 - 147 (1980).

3. M. Wijaya and E.C. Leong, Geotechnique, 67(7):637-645 (2017).

4. A.N. Zhou, D. Sheng and J.P. Carter, Geotechnique, 62(8):669-680 (2012).

5. A. Tarantino, Geotechnique, 59(9):751-762 (2009).

6. D.G. Fredlund and A. Xing, Canadian Geotechnical Journal, 31: 521 - 532 (1994).

7. Y. Klausner, Springer-Verlag, New York (1991).

8. J. Bear, New York: McGraw-Hill (McGraw-Hill Series in Water Resource and Environmental Eng), 567 pp (1979).

9. Y. Mualem, Water Resources Research, 10: 514 520 (1974).

10. J.-Y. Parlange, Water Resources Research, 12: 224 -228 (1976).

11. H.Q. Pham, D.G. Fredlund and S.L. Barbour, Géotechnique, 53: 293 - 298 (2003).

12. H.Q. Pham, D.G. Fredlund and S.L. Barbour, Canadian Geotechnical Journal, 42: 1548 - 1568 (2005).

13. Q. Zhai, H. Rahardjo and A. Satyanaga, Environmental Geotechnics, 6(4): 204-213 (2019).

14. Q. Zhai, H. Rahardjo, A. Satyanaga, G. Dai and Y.J. Du, Engineering Geology (Accepted in Apr 2020)

15. D.G. Fredlund and H. Rahardjo, John Wiley \& Sons, Inc. New York (1993).

16. C. W. W. Ng and B. Menzies, London: Taylor and Francis, 712 pp (2007).

17. E.C. Leong and H. Rahardjo, ASCE Journal of Geotechnical and Geoenvironmental Engineering, 123(12):1106-1117 (1997).

18. H. Yang, H. Rahardjo, E.C. Leong and D.G. Fredlund, Canadian Geotechnical Journal, 41: 908 920 (2004).

19. I.G.B. Indrawan, H. Rahardjo and E.C. Leong, Canadian Geotechnical Journal, 44: 20 - 32 (2007).

20. I. Meilani, H. Rahardjo and E.C. Leong, Canadian Geotechnical Journal, 42: 1509 - 1531 (2005).
21. M.T. Trinh, H. Rahardjo and E.C. Leong, Geotechnical Special Publication, 147: 1212 - 1222 (2006).

22. M. Dodge and C. Stinson, Microsoft Press, Washington, USA, 1088 pp (2007). 